BMJ Open Sport \& Exercise Medicine

\title{
Associations between altered movement patterns during single-leg squat and muscle activity at weight-transfer initiation in individuals with anterior cruciate ligament injury
}

\author{
Anna Trulsson, ${ }^{1,2}$ Michael Miller, ${ }^{3}$ Christina Gummesson, ${ }^{4}$ Martin Garwicz ${ }^{5}$
}

To cite: Trulsson A, Miller M, Gummesson C, et al. Associations between altered movement patterns during single-leg squat and muscle activity at weight-transfer initiation in individuals with anterior cruciate ligament injury. BMJ Open Sport Exerc Med 2016;2:e000131.

doi:10.1136/bmjsem-2016000131

- Prepublication history and additional material is available. To view please visit the journal (http://dx.doi.org/ 10.1136/bmjsem-2016000131)

CG and MG contributed equally.

Accepted 19 October 2016

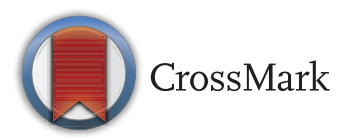

For numbered affiliations see end of article.

Correspondence to Dr Anna Trulsson; anna.trulsson@med.lu.se

\section{ABSTRACT}

Background: Little is known about factors contributing to the altered movement patterns observed in many individuals with anterior cruciate ligament (ACL) injury. We addressed whether altered muscular activity is such a factor.

Methods: 16 participants with unilateral, nonreconstructed ACL rupture were scored for altered movement patterns according to Test for Substitution Patterns (TSP), which includes the single-leg squat (SLS). Surface electromyography (SEMG), was recorded in the lower extremities at initiation of weighttransfer from double-leg to single-leg stance (eyes closed), simulating the initiation of an SLS. Normalised SEMG amplitudes 200-300 ms after weight-transfer initiation were compared between injured and noninjured sides, and correlated to the TSP scores for the SLS. Peak absolute SEMG amplitudes during 5 TSP test movements were also compared between sides.

Results: At weight-transfer initiation, muscle activity was lower in the tibialis anterior, gastrocnemius and peroneus longus muscles on the injured side. Low muscle activity correlated moderately to worse TSP scores for the SLS for the gluteus medius $\left(r_{s}=-0.56\right.$, $p=0.03)$, and gastrocnemius muscles $\left(r_{s}=-0.56\right.$, $\mathrm{p}=0.02$ ). Median peak absolute amplitude during TSP movements was lower in the quadriceps,

gastrocnemius and peroneus longus muscles on the injured side.

Conclusions: The altered patterns of muscle activity at weight-transfer initiation, correlations between lower activity at movement initiation and altered movement patterns during SLS and the altered peak amplitudes during TSP movements together indicate alterations in sensorimotor control that may contribute to the observed altered movement patterns. Future studies will determine if exercises targeting muscle activity initiation should complement customary ACL injury rehabilitation.

\section{INTRODUCTION}

In many individuals with anterior cruciate ligament (ACL) injury, altered movement

\section{What are the new findings?}

The findings indicate alterations in sensorimotor control that we suggest contribute to altered movement patterns in individuals with anterior cruciate ligament (ACL) injury and comprise:

- Lower muscle activity (surface electromyography) on the injured as compared with noninjured side in the shank muscles at movement initiation of a basic weight-transfer task (the start of the single-leg squat),

- Correlations between lower muscle activity at movement initiation in the gluteus medius and gastrocnemius muscles and more pronounced altered movement patterns during single-leg squat assessed with the observational Test for Substitution Patterns (TSP),

- Altered peak amplitudes in quadriceps, gastrocnemius and peroneus longus muscles on injured as compared with non-injured sides during TSP test movements.

How might it impact on clinical practice in the near future?

Rehabilitation targeting relearning of muscle activity initiation of muscles both proximal and distal to the knee joint might after additional investigation be advocated in addition to commonly used neuromuscular training following ACL injury.

patterns have been demonstrated during the performance of functional movements, ${ }^{1-3}$ and have been shown to be associated with, for example, knee osteoarthritis and injury mechanisms. ${ }^{4} 5$ Altered movement patterns, observed in non-injured individuals to a lesser extent, ${ }^{3}$ have been hypothesised to emerge from impaired sensorimotor control (the sensory, motor, and central integration and processing involved in maintaining joint 
stabilisation during movements) due to, for example, deviating muscular activity and/or on biomechanical instability. ${ }^{2} \quad 6 \quad 7$ Altered movement patterns are also assumed to be affected by decreased muscle force production, anticipation of instability or pain. However, the interplay between factors contributing to altered movement patterns is not well understood.

In individuals with ACL injury, inconsistent findings exist regarding patterns of muscular activity in the quadriceps and hamstrings muscles. ${ }^{8}{ }^{9}$ In the shank, earlier onset $^{10}$ and decreased activity in the gastrocnemius muscles have been described during gait in individuals with ACL injury. ${ }^{8}$ Both timing and degree of muscle activity have been studied, ${ }^{1112}$ and Kim et al ${ }^{11}$ concluded that the magnitude of muscular activity at movement initiation during transition from double-leg to single-leg stance was more important for controlling knee and pelvic stability than activity onset in non-injured individuals. Notably, both delay of muscular activity and affected recruitment order have been interpreted as indications of altered sensorimotor control that could affect the individual's activity level and performance. ${ }^{13-16}$ Also, in a recent report by our group, decreased muscular activity was found to correlate to specific, unfavourable altered movement patterns during maximum knee flexion of single-leg squats (SLS) in individuals with ACL injury $^{17}$ when using an observational test of common rehabilitation movements with varying degree of difficulty (Test for Substitution Patterns, TSP ${ }^{318}$ ) and surface electromyography (SEMG). In the present study, we extended this investigation to an assessment of the magnitude of muscle activity at the very start of the SLS- the weight transfer from double-leg to single-leg stance.

Muscular activity during dynamic knee stabilisation ${ }^{13}$ (synonymous with functional knee stability ${ }^{6}$ ) is not mainly determined by feedback but even more importantly by feedforward mechanisms involved in anticipatory muscular activity. ${ }^{6}{ }^{7}{ }^{19}$ This emphasises the importance of muscular activity at movement initiation, and previous studies have shown that timing and magnitude of muscle activity is of importance in movement initiation. ${ }^{11} 16 \quad 20$ However, associations between initial muscular activity and altered movement patterns in individuals with ACL injury have, to the best of our knowledge, not been investigated. Therefore, to further investigate factors that may contribute to altered movement patterns, we assessed muscular activity at weighttransfer initiation from double-leg to single-leg stance simulating the start of the SLS, and scored altered movement patterns observed during the performance of SLS and also four other test movements included in the TSP. The complete TSP was used to compare possible overall changes in SEMG activity between the injured and noninjured sides during five test movements with varying difficulty that are often used in ACL rehabilitation, and the weight-transfer task was chosen since it constitutes the start of the SLS, and also because it entails fundamental strategies for other, more complex movements.
The main aims of the present study were to (1) describe and compare electromyographic amplitudes in six muscles of the hip, thigh and shank on the injured and non-injured sides at weight-transfer initiation in individuals with altered movement patterns and a unilateral, total ACL rupture, and to (2) characterise associations between specific altered movement patterns during SLS and deviations in muscular activity at weight-transfer initiation. To investigate possible overall changes in neuromuscular activity, we also (3) compared the peak absolute amplitude between the injured and non-injured sides for each muscle recorded during the weighttransfer task and during five TSP test movements of varying difficulty. ${ }^{3} 18$

\section{MATERIALS AND METHODS}

For details, see the online supplementary material.

\section{Participants}

A total of 16 participants, 10 women, aged 19-48 years (mean 29.5, SD 9.5), volunteered to participate. All had a total, unilateral, non-reconstructed ACL rupture sustained at mean 3.6 months (2-11 months, SD 2.3) before testing with increased sagittal laxity verified by an orthopaedic surgeon and with MRI and/or arthroscopy. Participants with knee pain in daily life or injury to the contralateral knee were not included. All participants gave written, informed consent, and the study was approved by the Regional Ethical Review Board in Lund, Sweden, Dnr 2010/387. For details, see online supplementary material and Trulsson et al. ${ }^{17}$

\section{Settings and procedure}

First, the participants performed the five TSP test movements (see below) and then the weight-transfer task from double-leg to single-leg stance with eyes closed, three trials for each movement while the SEMG was recorded, synchronised with an electrogoniometer and a video camera.

\section{Test movements and TSP scoring}

The complete manual of the TSP has been described previously, ${ }^{3}{ }^{18}$ and a detailed description as performed in the present study can be found in ref. ${ }^{17}$. In brief, the TSP consists of five test movements-SLS, double-leg squat, forward lunge, tip-toe standing knee flexion and body weight altering, during which 18 specific unfavourable altered movement patterns are scored using a fourpoint, ordinal scale $(0-3)$. In the present study, the score for the test movement SLS was used in the calculations of correlation between altered movement patterns and initial muscle activity. In the SLS movement, the following altered movements were scored: 'knee medial to supporting foot', 'pronation of foot', 'lateral displacement of hip-pelvis region', 'displacement of trunk'. The TSP score for the SLS movement could vary from 0 to a maximum of 12 and median score on the non-injured 
side was 0 (minimum-maximum $0-3$ ) and on the injured side was 2 (minimum-maximum 0-6; $\mathrm{p}=0.003$ ). The most frequent substitution patterns in SLS were 'knee medial to the supporting foot' and 'displacement of trunk' (both present in 9/16 participants).

\section{Weight-transfer task}

During the weight-transfer task from double-leg to singleleg stance, standardised verbal and visual instructions according to the manual were given. The task was performed with eyes closed as in investigations. ${ }^{21} 22$ The participant was instructed to stand with equal weight on both legs, look at a fixed point on the opposite wall and then close the eyes. The examiner gave distinct verbal instructions to transfer weight to stand on one leg for about $2 \mathrm{~s}$. Thereafter, the participant returned to two-leg stance for about $5 \mathrm{~s}$ before receiving a new instruction. Three consecutive trials were performed, first standing three times on the non-injured leg and then on the injured leg similarly to previous analogous investigations. ${ }^{23}$

\section{SEMG, electrogoniometers and video camera}

For details including piloting and protocol development, see online supplementary material and ref. 17. In short, the SEMG was recorded bilaterally from gluteus medius, biceps femoris (long head), quadriceps femoris vastus lateralis, tibialis anterior, medial head of gastrocnemius and the peroneus longus muscle. The Mega Win Software V.3.1 was used to digitally filter the raw SEMG signals with a band-pass filter with cut-off frequencies of 30 and $400 \mathrm{~Hz}$ and epochs of $125 \mathrm{~ms}$ to calculate the root mean square value. Surface electrodes were placed according to SENIAM recommendations. ${ }^{24}$ Strain gauge electrogoniometers (SG150, Biometrics, Newport, UK), were used to measure knee flexion/extension angle, and were mounted laterally on the knees. The goniometer signals were recorded on the same data logger as the SEMG signal. The video camera captured the session from the front and was synchronised with the SEMG and electrogoniometer, so that the movements could also be assessed afterwards.

\section{Data analysis and statistics \\ Data analysis}

The same examiner observed and scored all participants according to the protocol. The examiner identified a trial during which the participant was stable during two-leg stance (=stood with good balance and no observable movement to support balance) at transfer from double-leg to single-leg stance. To analyse SEMG activity during the chosen trial, the Mega Win software was used to present the root mean square averaged SEMG amplitudes (microvolts, $\mu \mathrm{V}$ ) on a time axis (milliseconds, ms). A baseline was identified as the moment with $0^{\circ}$ of change in electrogoniometer measurement and with minimal changes in SEMG. The start of the weight transfer was identified as the instance when the electrogoniometer changed $1-2^{\circ}$ of knee extension of the leg the participant was about to transfer the weight to. The mean SEMG amplitude in $\mu \mathrm{V}$, during $100 \mathrm{~ms}$, starting at $200 \mathrm{~ms}$ (in analogy with ${ }^{15}$ ) after the start of the weight transfer was recorded for each muscle, each leg and each individual. To normalise the mean amplitudes during the $100 \mathrm{~ms}$ period, the highest amplitude during the test movements as a whole for each muscle, each leg for each individual was calculated as a ratio of the mean amplitude to the highest amplitude. These normalised values were then used in the statistical analysis.

\section{Statistics}

The Wilcoxon signed-rank test was used to test for differences between sides with respect to TSP scores and normalised SEMG amplitudes. Differences $\mathrm{p} \leq 0.05$ were considered statistically significant. The non-parametric CI $(95 \%)$ was calculated for the median of the paired differences. ${ }^{25}$ For the correlation calculations between the normalised SEMG amplitude and the results of the TSP score, the Spearman's correlation coefficients $\left(r_{s}\right)$ were used. All calculations and statistical analyses were carried out using SPSS V.11.5 and IBM SPSS Statistics V.20.0.

\section{RESULTS}

\section{Muscular activity at movement initiation}

At movement initiation, $200 \mathrm{~ms}$ after the identified weight transfer from double-leg to single-leg stance, three of the six muscles displayed lower SEMG activity on the injured compared with the non-injured side. The median difference in normalised amplitude between the injured and non-injured sides was 0.02 (95\% CI 0.00 to $0.13 ; \mathrm{p}=0.003$ ) for tibialis anterior, 0.05 (95\% CI 0.00 to $0.10 ; \mathrm{p}=0.003)$ for gastrocnemius medialis and 0.05 (95\% CI 0.00 to $0.16 ; \mathrm{p}=0.034$ ) for the peroneus longus muscle (table 1).

\section{Correlations between SEMG and TSP scores}

During the weight-transfer task, muscle activity of the gluteus medius and the gastrocnemius medialis were negatively correlated to the TSP score for the SLS movement, with $\mathrm{r}_{\mathrm{s}}=-0.56, \mathrm{p}=0.03(95 \%$ CI -0.82 to -0.08$)$ and $r_{s}=-0.56, p=0.02(95 \%$ CI -0.83 to -0.09$)$, respectively. This indicates that the lower the activity in these muscles at weight-transfer initiation, the more pronounced is the altered movement patterns in the SLS. No other correlations between deviations in muscle activity and TSP scores for the SLS were found, either on the injured or on the non-injured side.

\section{Peak SEMG activity during the test movements}

The peak absolute SEMG amplitude in $\mu \mathrm{V}$, recorded during the five test movements was lower for the quadriceps, gastrocnemius and peroneus longus muscles on the injured compared with the non-injured side. For the quadriceps, gastrocnemius and peroneus longus, the median differences between the injured and the noninjured sides were 80.0 (95\% CI 7.0 to $137.0 ; \mathrm{p}=0.013$ ), 
Table 1 Median surface electromyography normalised amplitude at movement initiation, quartiles, $p$ value and median difference $(95 \% \mathrm{Cl})$ for injured and non-injured sides for the weight-transfer task from double-leg to single-leg stance for each muscle are presented $(\mathrm{N}=16)$

\begin{tabular}{llllc}
\hline Muscle & $\begin{array}{l}\text { Median amplitude } \\
\mathbf{( Q 1}, \mathbf{Q 3}) \text { non-injured side }\end{array}$ & $\begin{array}{l}\text { Median amplitude } \\
(\mathbf{Q 1}, \mathbf{Q 3}) \text { injured side }\end{array}$ & $\begin{array}{l}\text { p Value } \\
\text { (95\% CI) }\end{array}$ & $\begin{array}{l}\text { Median difference } \\
(95 \%\end{array}$ \\
\hline Gluteus medius & $0.17(0.10,0.21)$ & $0.08(0.04,0.18)$ & 0.056 & $0.05(0.00$ to 0.11$)$ \\
Biceps femoris & $0.05(0.03,0.06)$ & $0.07(0.04,0.10)$ & 0.438 & $-0.005(-0.06$ to 0.02$)$ \\
Quadriceps, vastus lateralis & $0.04(0.03,0.07)$ & $0.04(0.01,0.06)$ & 0.469 & $0.00(-0.01$ to 0.02$)$ \\
Tibialis anterior & $0.04(0.02,0.12)$ & $0.01(0.01,0.03)$ & 0.003 & $0.02(0.00$ to 0.13$)$ \\
Gastrocnemius medialis & $0.09(0.05,0.16)$ & $0.05(0.02,0.11)$ & 0.003 & $0.05(0.00$ to 0.10$)$ \\
Peroneus longus & $0.13(0.05,0.17)$ & $0.04(0.02,0.13)$ & 0.034 & $0.05(0.00$ to 0.16$)$ \\
\hline
\end{tabular}

Q1, Q3=quartiles 1 (25th percentile) and 3 (75th percentile).

$\mathrm{p}$ Value, Wilcoxon signed-rank test.

$47.0(95 \%$ CI 5.0 to $117.0 ; \mathrm{p}=0.034)$ and 94.0 (95\% CI -18.0 to 203.0; $\mathrm{p}=0.030$ ), respectively. For the gluteus medius, biceps femoris and tibialis anterior muscles, no differences in peak absolute SEMG amplitude during the test movements were found (table 2).

\section{DISCUSSION}

The main findings in the present study were the (1) correlations between low SEMG activity in the gluteus medius and gastrocnemius muscles at weight-transfer initiation and more pronounced altered movement patterns during SLS on injured side, (2) altered patterns of muscle activity at weight-transfer initiation in shank muscles and altered peak amplitudes during TSP movements in quadriceps, gastrocnemius and peroneus longus muscles on injured side. Taken together, these findings indicate alterations in sensorimotor control that we suggest may contribute to the altered movement patterns observed in these individuals.

\section{Correlations between SEMG and TSP scores}

The correlations between low muscle activity in gluteus medius at movement initiation and more pronounced specific altered movement patterns during SLS on the injured side (with the pattern 'knee medial to the supporting foot' as one of the most frequent) is in accordance with the results by Kim et $a l^{11}$ who showed that the magnitude of muscular activity in gluteus medius at movement initiation (anticipatory activation) was of relevance for the reduction of 'knee medial to supporting foot' and for preventing 'lateral displacement of hip-pelvis region' in non-injured individuals.

The correlation between low muscle activity at weight-transfer initiation in the gluteus medius and gastrocnemius muscles and the scores of altered movement patterns of SLS, suggest that the lower the initial muscular activity at transfer initiation, the more altered movement patterns or more pronounced altered movement patterns were observed later in SLS movement. These muscles play a crucial role in weight bearing, but it is also likely that additional alterations can emerge later during movement contributing to the observed altered movement patterns. Support for this was found in a recent study, where lower muscle activity in, for example, gluteus medius at maximum knee flexion during SLS correlated to a more pronounced medial knee displacement. ${ }^{17}$

Muscular activity at movement initiation and in peak SEMG activity-implications of altered sensorimotor control and possible relevance to clinical implications In studies of patterns of muscle activity, the assessment of onset time is often used, where delayed onset has been interpreted as an indication of deficiency or

Table 2 Median peak absolute amplitudes in $\mu \mathrm{V}$ for each muscle during any of the five test movements for the 16 participants with anterior cruciate ligament rupture

\begin{tabular}{llllr}
\hline Muscle & $\begin{array}{l}\text { Median peak amplitude } \\
(\mathbf{Q 1}, \mathbf{Q 3}) \text { non-injured side }\end{array}$ & $\begin{array}{l}\text { Median peak amplitude } \\
(\mathbf{Q 1}, \mathbf{Q 3}) \text { injured side }\end{array}$ & $\begin{array}{l}\text { p Value } \\
\text { 95\% Cl }\end{array}$ \\
\hline Gluteus medius & $112.0(76.8,151.5)$ & $102.0(79.8,154.2)$ & 0.266 & $12.5(-11.0$ to 34.0) \\
Biceps femoris & $124.5(70.8,152.2)$ & $135.5(98.8,158.0)$ & 0.234 & $-16.0(-41.0$ to 33.0) \\
Quadriceps, vastus lateralis & $301.0(230.8,351.0)$ & $220.5(149.5,304.0)$ & 0.013 & $80.0(7.0$ to 137.0$)$ \\
Tibialis anterior & $490.5(362.2,608.5)$ & $441.5(309.5,533.0)$ & 0.079 & $78.0(-22.0$ to 153.0) \\
Gastrocnemius medialis & $344.5(300.0,372.0)$ & $282.5(232.5,347.5)$ & 0.034 & $47.0(5.0$ to 117.0) \\
Peroneus longus & $330.0(241.5,492.5)$ & $243.5(215.8,310.5)$ & 0.030 & $94.0(-18.0$ to 203.0) \\
\hline
\end{tabular}

Q1, Q3=quartiles 1 (25th percentile) and 3 (75th percentile).

$\mathrm{p}$ Value, Wilcoxon signed-rank test. 
decreased sensorimotor control. ${ }^{11}{ }^{13-15}$ Similarly, lower amplitudes in the gastrocnemius muscles, also found in the present study, have previously been found in individuals with ACL injury. ${ }^{8}$ Although the same assessments were not used, the lower amplitude at weight-transfer initiation in distal muscles in the present study might be put into the context of the results of Dingenen $e t a l,{ }^{16}$ describing delayed onset in the gastrocnemius and tibialis anterior muscles when comparing individuals with ACL injury to controls performing a weight-transfer task. However, no differences were found by Dingenen $e t$ al in the peroneus longus muscle. The relevance of distal muscle activity to ACL injury and associations between ACL injury and, for example, the gastrocnemius activity is until now not clear ${ }^{26} 27$ and contradictory results describing both increased and decreased activity have been found. ${ }^{8}{ }^{10}$ Interestingly, during a similar transfer task to the one in the present study, participants with chronic ankle instability were found to have significantly later onset for the same shank muscles as in the present study, and also for the hamstrings, tensor fascia latae and gluteus medius muscles but not the quadriceps, compared with controls, ${ }^{28}$ posing a question for future studies if altered patterns of muscle activity might not be related to a specific ligament injury, but rather to, alterations in sensorimotor control.

The lower median peak absolute amplitude in the quadriceps, gastrocnemius and peroneus longus muscles during TSP test movements in the present study should be interpreted with some caution. One explanation for the lower absolute amplitude could be muscle weakness due to inadequate central drive on motor unit recruitment and $\alpha$ motor neuron firing frequency modulation in the injured side, since lower muscle force production is well documented in thigh muscles after ACL injury. ${ }^{29}{ }^{30}$ Nevertheless, the fact that there was not a reduction of peak absolute amplitude of all muscles indicates altered patterns of muscular activity and thus altered sensorimotor control.

After ACL injury, the sensorimotor control is affected by alterations in afferent proprioceptive input, altered information processing and maladaptive efferent motor commands resulting in altered muscle activation. ${ }^{2} 61931$ The alterations in muscular activity found in the present study could therefore be argued to be a manifestation of impaired sensorimotor control affecting performance and to be one mechanism underlying altered movement patterns. Notably, anticipatory muscular activity has been shown to be of importance in joint stabilisation strategies, ${ }^{32}$ and individuals with ACL injury who compensate well for their injury (copers) have been shown to develop compensation strategies not only related to muscular strength but also to the tuning of muscle activation when relearning motor control. ${ }^{133} 34$ Therefore, the findings of altered muscle activity and altered movement patterns in the present study could be of potential value for the design of ACL rehabilitation and should be studied further. Such rehabilitation, aiming at relearning of stabilising postural activity, has been described and evaluated with good results 15 years after initial injury in individuals with ACL injury by Zätterström et $a l^{35-38}$ and favourable results have been obtained also with perturbation training eliciting dynamic joint stabilisation by muscular co-activation of the lower extremity. ${ }^{39}$

\section{Limitations}

Some limitations should be considered when interpreting the present results. To be able to compare SEMG amplitudes between individuals or muscles, a normalisation calculation is often performed, using maximal voluntary contraction (MVC). Owing to the relatively short time since initial ACL injury, we made the judgement that there was the risk that the strain during MVCs could endanger the injured knee and cause a new knee injury and/or subsequent transient pain also affecting the assessment. We chose therefore to use peak absolute amplitudes for each muscle for the same leg produced during the TSP test movements for normalisation calculations. ${ }^{40} 41$

The use of comparison between left and right sides can constitute a possible limitation. But, since there are considerable interindividual variations in the performance of the same movement, it is difficult to deduce whether altered muscle activity underlies altered movement patterns unless the patient is his/her own control. Also, side-to-side differences were not found for SEMG temporal measures among non-injured controls, for example, medial gastrocnemius during single-leg hop, a more strenuous activity than the one performed here ${ }^{1526}$ Also, no differences between sides were found in onset times in controls performing an almost identical task as in the present study, except for in the gluteus maximus muscle. ${ }^{16}$ It is further known that deficits in proprioception and postural control are evident also in the noninjured side after ACL injury. ${ }^{42}$ If this is the case in this study, it is possible that even more pronounced differences would be found if data from the present study could be compared with the data of non-injured controls.

Another issue is that the order of testing was not randomised. This could result in a learning effect where the task on the injured side, performed after the noninjured side, was easier. If this was the case, a randomisation of the test order most likely would result in even more pronounced differences between sides.

The present study was a first step towards understanding associations between muscle activity at movement initiation and altered movement patterns. More studies are needed to further the understanding and to establish clinical guidelines. However, the physiological differences between sides in our study do exist, and it cannot be excluded that they could affect function and/or be of potential diagnostic value in ACL rehabilitation.

\section{CONCLUSIONS}

Altered patterns in muscular activity shown as lower amplitude at weight-transfer initiation in shank muscles 
during weight transfer but also as lower peak absolute amplitude partly in other muscles (the quadriceps, gastrocnemius and peroneus longus muscles) during five TSP test movements suggest alterations in sensorimotor control. This is further supported by correlations between lower muscle activity at weight transfer in gluteus medius and gastrocnemius muscles and altered movement patterns. It is suggested that the alterations in sensorimotor control found here may contribute to altered movement patterns, but further investigations of contributing factors are warranted. All in all, future studies will show whether exercises targeting relearning of motor control strategies and aberrant muscle activity initiation in muscles both proximal and distal to the knee joint should be advocated as a complement to customary ACL injury rehabilitation.

\section{Author affiliations}

${ }^{1}$ Department of Health Sciences, Physiotherapy, Lund University, Lund, Sweden

${ }^{2}$ Department of Pain Rehabilitation, Skåne University Hospital, Lund, Sweden

${ }^{3}$ Department of Health Sciences, Physiotherapy, Lund University, Lund, Sweden

${ }^{4}$ Faculty of Medicine, Center for Teaching and Learning, Lund University, Lund, Sweden

${ }^{5}$ Department of Experimental Medical Science, Neuronano Research Center. Lund University, Lund, Sweden

Acknowledgements The authors would kindly like to acknowledge Professor Jonas Björk, Division of Occupational and Environmental Medicine at Lund University, for statistical consultation, RPT Örjan Sundewall, Department of Orthopedics at Skane University Hospital for participant management, EE, PhD Gert-Åke Hansson, Occupational and Environmental Medicine, Lund University and University and Regional Laboratories Region Scania, Lund, for expertise on SEMG, and the Department of Health Sciences, Division of Physiotherapy, Lund University, Sweden for providing the equipment.

Contributors AT, CG and MG provided the concept, idea and fund procurement, and research design. AT, MM and CG provided the data collection. AT performed the data analysis and the statistical analysis in consultation with Professor Jonas Björk. AT was mainly responsible for the writing, while AT, CG and MG drafted the manuscript and all authors read and approved the final version. AT provided project management and the Department of Health Sciences, Division of Physiotherapy, Lund University provided the equipment.

Funding This study was funded by the Swedish Research Council, project number 14015 (principal investigator MG), Swedish National Centre for Research in Sports, Medical Faculty of Lund University, Sweden, Region Skane, Sweden, Ann-Mari och Ragnar Hemborgs Minnesfond, Lund University, Sweden, Emy Thulins Forskningsfond, Lund University, Sweden and Professor Stig Radners fond, Lund University, Sweden.

Competing interests None declared.

Patient consent Obtained.

Ethics approval Regional Ethical Review Board in Lund, Sweden, Dnr 2010/387.

Provenance and peer review Not commissioned; externally peer reviewed.

Open Access This is an Open Access article distributed in accordance with the Creative Commons Attribution Non Commercial (CC BY-NC 4.0) license, which permits others to distribute, remix, adapt, build upon this work noncommercially, and license their derivative works on different terms, provided the original work is properly cited and the use is non-commercial. See: http:// creativecommons.org/licenses/by-nc/4.0/

\section{REFERENCES}

1. Rudolph KS, Snyder-Mackler L. Effect of dynamic stability on a step task in ACL deficient individuals. J Electromyogr Kinesiol 2004;14:565-75.

2. Ingersoll CD, Grindstaff TL, Pietrosimone BG, et al. Neuromuscular consequences of anterior cruciate ligament injury. Clin Sports Med 2008;27:383-404. vii.

3. Trulsson A, Garwicz M, Ageberg E. Postural orientation in subjects with anterior cruciate ligament injury: development and first evaluation of a new observational test battery. Knee Surg Sports Traumatol Arthrosc 2010;18:814-23.

4. Tanamas S, Hanna FS, Cicuttini FM, et al. Does knee malalignment increase the risk of development and progression of knee osteoarthritis? A systematic review. Arthritis Rheum 2009;61:459-67.

5. Hewett TE, Torg JS, Boden BP. Video analysis of trunk and knee motion during non-contact anterior cruciate ligament injury in female athletes: lateral trunk and knee abduction motion are combined components of the injury mechanism. Br J Sports Med 2009;43:417-22.

6. Riemann BL, Lephart SM. The sensorimotor system, part I: the physiologic basis of functional joint stability. J Athl Train 2002;37:71-9.

7. Ward S, Pearce AJ, Pietrosimone B, et al. Neuromuscular deficits after peripheral joint injury: a neurophysiological hypothesis. Muscle Nerve 2015;51:327-32

8. Papadonikolakis A, Cooper L, Stergiou N, et al. Compensatory mechanisms in anterior cruciate ligament deficiency. Knee Surg Sports Traumatol Arthrosc 2003;11:235-43.

9. Demont RG, Lephart SM, Giraldo JL, et al. Muscle preactivity of anterior cruciate ligament-deficient and -reconstructed females during functional activities. J Athl Train 1999;34:115-20.

10. Lindström M, Felländer-Tsai L, Wredmark T, et al. Adaptations of gait and muscle activation in chronic ACL deficiency. Knee Surg Sports Traumatol Arthrosc 2010;18:106-14

11. Kim D, Unger J, Lanovaz JL, et al. The Relationship of anticipatory gluteus medius activity to pelvic and knee stability in the transition to single-leg stance. PM R 2015;8:138-44.

12. Medina JM, Valovich McLeod TC, Howell SK, et al. Timing of neuromuscular activation of the quadriceps and hamstrings prior to landing in high school male athletes, female athletes, and female non-athletes. J Electromyogr Kinesiol 2008;18:591-7.

13. Wikstrom EA, Tillman MD, Chmielewski TL, et al. Measurement and evaluation of dynamic joint stability of the knee and ankle after injury. Sports Med 2006;36:393-410.

14. Kaneko F, Onari K, Kawaguchi K, et al. Electromechanical delay after ACL reconstruction: an innovative method for investigating central and peripheral contributions. J Orthop Sports Phys Ther 2002;32:158-65.

15. Wojtys EM, Huston LJ. Neuromuscular performance in normal and anterior cruciate ligament- deficient lower extremities. Am J Sports Med 1994;22:89-104.

16. Dingenen B, Janssens L, Luyckx $\mathrm{T}$, et al. Lower extremity muscle activation onset times during the transition from double-leg stance to single-leg stance in anterior cruciate ligament injured subjects. Hum Mov Sci 2015;44:234-45

17. Trulsson A, Miller M, Hansson G-Å, et al. Altered movement patterns and muscular activity during single and double leg squats in individuals with anterior cruciate ligament injury. BMC Musculoskele Disord 2015;16:28.

18. Trulsson A, Roos EM, Ageberg E, et al. Relationships between postural orientation and self reported function, hop performance and muscle power in subjects with anterior cruciate ligament injury. BMC Musculoskelet Disord 2010;11:143.

19. Riemann BL, Lephart SM. The sensorimotor system, part ii: the role of proprioception in motor control and functional joint stability. $J$ Athl Train 2002;37:80-4

20. Rudolph KS, Eastlack ME, Axe MJ, et al. 1998 Basmajian Student Award Paper: movement patterns after anterior cruciate ligament injury: a comparison of patients who compensate well for the injury and those who require operative stabilization. $J$ Electromyogr Kinesiol 1998;8:349-62.

21. Okuda K, Abe N, Katayama Y, et al. Effect of vision on postural sway in anterior cruciate ligament injured knees. J Orthop Sci 2005;10:277-83.

22. Dingenen B, Staes FF, Janssens L. A new method to analyze postural stability during a transition task from double-leg stance to single-leg stance. J Biomech 2013;46:2213-9.

23. Dingenen $B$, Janssens $L$, Luyckx $T$, et al. Postural stability during the transition from double-leg stance to single-leg stance in anterior 
cruciate ligament injured subjects. Clin Biomech (Bristol, Avon) $2015 ; 30: 283-9$.

24. SENIAM. Surface Electromyography for the Non-Invasive Assessment of Muscles. 2016. http://seniam.org/sensor_location. $\mathrm{htm}$. (accessed 20160221, 2016).

25. Hollander M, Wolfe D. Non-parametric statistical methods. 2nd edn. New York: John Wiley \& Sons Inc. 1999.

26. Klyne DM, Keays SL, Bullock-Saxton JE, et al. The effect of anterior cruciate ligament rupture on the timing and amplitude of gastrocnemius muscle activation: a study of alterations in EMG measures and their relationship to knee joint stability. $J$ Electromyogr Kinesiol 2012;22:446-55

27. Shanbehzadeh S, Mohseni Bandpei MA, Ehsani F. Knee muscle activity during gait in patients with anterior cruciate ligament injury: a systematic review of electromyographic studies. Knee Surg Sports Traumatol Arthrosc doi:10.1007/s00167-015-3925-9. 2015: Dec 24 [Epub ahead of print].

28. Van Deun S, Staes FF, Stappaerts KH, et al. Relationship of chronic ankle instability to muscle activation patterns during the transition from double-leg to single-leg stance. Am J Sports Med 2007;35:274-81.

29. Tsepis E, Vagenas G, Ristanis $\mathrm{S}$, et al. Thigh muscle weakness in ACL-deficient knees persists without structured rehabilitation. Clin Orthop Relat Res 2006;450:211-18.

30. Failla MJ, Arundale AJ, Logerstedt DS, et al. Controversies in knee rehabilitation: anterior cruciate ligament injury. Clin Sports Med 2015;34:301-12.

31. Lephart S, Fu F. Proprioception and neuromuscular control in joint stability. In: Lephart S, Fu F, eds. Proprioception and neuromuscular control in joint stability. Human Kinetics. 2000:5-29, 77-88, 171-96.

32. Williams GN, Chmielewski T, Rudolph K, et al. Dynamic knee stability: current theory and implications for clinicians and scientists. J Orthop Sports Phys Ther 2001;31:546-66.

33. Mulder T, Hulstyn W. Sensory feedback therapy and theoretical knowledge of motor control and learning. Am J Phys Med 1984;63:226-44.
34. Pietrosimone B, Blackburn JT, Harkey MS, et al. Clinical strategies for addressing muscle weakness following knee injury. Clin Sports Med 2015;34:285-300.

35. Zätterström R, Fridén $\mathrm{T}$, Lindstrand $\mathrm{A}$, et al. Rehabilitation following acute anterior cruciate ligament injuries-a 12 month follow-up of a randomized clinical trial. Scand J Med Sci Sports 2000;10:156-63.

36. Zätterström R, Fridén $\mathrm{T}$, Lindstrand $\mathrm{A}$, et al. Early rehabilitation of acute anterior cruciate ligament injury--a randomized clinical trial. Scand J Med Sci Sports 1998;8:154-9.

37. Ageberg E, Pettersson A, Fridén T. 15-year follow-up of neuromuscular function in patients with unilateral nonreconstructed anterior cruciate ligament injury initially treated with rehabilitation and activity modification: a longitudinal prospective study. Am J Sports Med 2007;35:2109-17.

38. Neuman P, Englund M, Kostogiannis I, et al. Prevalence of tibiofemoral osteoarthritis 15 years after nonoperative treatment of anterior cruciate ligament injury: a prospective cohort study. Am $J$ Sports Med 2008;36:1717-25.

39. Di Stasi SL, Snyder-Mackler L. The effects of neuromuscular training on the gait patterns of ACL-deficient men and women. Clin Biomech (Bristol, Avon) 2012;27:360-5.

40. Burden A. How should we normalize electromyograms obtained from healthy participants? What we have learned from over 25 years of research. J Electromyogr Kinesiol 2010;20:1023-35.

41. Sousa ASP, Tavares JMRS. Surface electromyographic amplitude normalization methods: a review. In: Hiroki Takada, (Graduate School of Engineering UoF, Japan), ed. Electromyography: new developments, procedures and applications. Neuroscience Research Progress, Nova Science Publishers, Inc. 2012: 85-101.

42. Negahban $\mathrm{H}$, Mazaheri M, Kingma I, et al. A systematic review of postural control during single-leg stance in patients with untreated anterior cruciate ligament injury. Knee Surg Sports Traumatol Arthrosc 2014;22:1491-504. 